\title{
Inelastic neutron scattering study of light-induced dynamics of a photosynthetic membrane system
}

\author{
A. Furrer and A. Stöckli \\ Laboratory for Neutron Scattering, ETH Zürich and PSI Villigen, CH-5232 Villigen PSI, Switzerland \\ (Received 22 June 2009; revised manuscript received 19 November 2009; published 5 January 2010)
}

\begin{abstract}
Inelastic neutron scattering was employed to study photoeffects on the molecular dynamics of membranes of the photosynthetic bacterium Rhodopseudomonas viridis. The main photoactive parts of this biomolecular system are the chlorophyll molecules whose dynamics were found to be affected under illumination by visible light in a twofold manner. First, vibrational modes are excited at energies of 12(2) and $88(21) \mathrm{cm}^{-1}$. Second, a partial "freezing" of rotational modes is observed at energies of 1.2(3) and 2.9(5) $\mathrm{cm}^{-1}$. These results are attributed to a possible coupling between molecular motions and particular mechanisms in the photosynthetic process.
\end{abstract}

DOI: 10.1103/PhysRevE.81.011901

PACS number(s): 87.14.ep, 87.15.H-, 87.64.Bx

\section{INTRODUCTION}

Photosynthesis, i.e., the process by which chlorophyllprotein complexes convert light energy to chemical energy, is one of the most important biological processes as it provides energy, food, and oxygen for all heterotrophic organisms. Most of the chlorophylls and other pigments in membranes of green plants and bacteria function as molecular antennas absorbing solar energy and conveying it to the specialized chlorophylls of the reaction center. When a chlorophyll molecule of an antenna complex absorbs light of a particular wavelength, it is electronically excited. It may either loose the excitation energy to a chlorophyll molecule of the reaction center and thereby realize a photochemical energy transfer or return to its ground state through relaxation mechanisms. The latter can be either radiative, in which case fluorescence occurs (i.e., a photon is emitted), or radiationless, resulting in the excitation of lattice modes. The lightharvesting power of the chromophores is the result of molecular orientation within the membrane, but its efficiency is dynamically disturbed by their rotational degrees of freedom, particularly at physiological temperatures. However, the detrimental effect of the Brownian motion may be hindered under illumination by light [1].

Spectroscopic investigations of photoeffects on the dynamical properties of the chlorophyll molecules are therefore essential toward a more detailed understanding of photosynthesis. The presently available experimental information, however, is rather scarce. This is certainly due to the structural complexity of these biomolecular systems, which makes a proper identification and interpretation of experimental data difficult and often arbitrary. What is needed is a selective labeling of the constituents of interest which can be achieved, for instance, in neutron spectroscopic measurements by contrast variation through partial deuteration of the protonic sites [2]. In the present inelastic neutron scattering (INS) experiments we used the photoactivity of the chlorophyll molecules to achieve this selection. Similar experiments were recently performed for demonstrating the structure-dynamics-function relationship during the photocycle of bacteriorhodopsin [3].

In INS experiments the incident neutrons lose or gain energy as a result of interactions with the external and internal lattice modes associated with all the constituents of a biomolecular sample. The external modes may be either translational or rotational, in which case a larger building block of the biomolecular system (e.g., a chlorophyll molecule) moves as an approximately rigid entity, whereas the internal modes correspond to vibrations, i.e., intramolecular dynamical deformations within the above subunits. The energies associated with the external modes are in the range of typically $1-100 \mathrm{~cm}^{-1}$, depending on the size of the building blocks, whereas the internal modes may extend up to a few hundreds of $\mathrm{cm}^{-1}$. In the case of organic molecules the signal is dominated by the incoherent scattering of the hydrogen nuclei. For proteins in solution extensive scattering from the buffer can be avoided by using buffers made from $\mathrm{D}_{2} \mathrm{O}$ [4].

Here, we report on the light-induced molecular dynamics of membranes of the photosynthetic bacterium Rhodopseudomonas viridis observed by INS experiments. Details of the experimental procedure are described in Sec. II. The results and the data analysis are presented in Sec. III, followed by a discussion and some conclusions in Secs. IV and $\mathrm{V}$, respectively.

\section{EXPERIMENT}

A suspension of membranes of the photosynthetic bacterium Rhodopseudomonas viridis in $\mathrm{D}_{2} \mathrm{O}$ buffer at a protein concentration of $20 \mathrm{mg} / \mathrm{ml}$ was prepared according to Ref. [5]. The membranes consist of a regular array of structural units. Each unit is composed of a central core (containing the reaction center complex) surrounded by a subdivided ring of protein (of likely antenna function) as demonstrated by electron microscopy [6]. The structural features of the photosynthetic reaction center were elucidated in great detail by $\mathrm{x}$-ray crystallographic analysis [7]. In order to identify the individual structural units, we measured the absorption spectrum which exhibits a broad maximum at $1010 \mathrm{~nm}$ [with full width at half maximum $(\mathrm{FWHM}) \approx 100 \mathrm{~nm}]$ and smaller peaks at 820 and $600 \mathrm{~nm}(\mathrm{FWHM} \approx 30 \mathrm{~nm})$ due to bacteriochlorophyll $\mathrm{b}$ from the light-harvesting complex and from the reaction center, respectively. Further peaks below $500 \mathrm{~nm}$ correspond to cytochromes and carotenoids. These assign- 


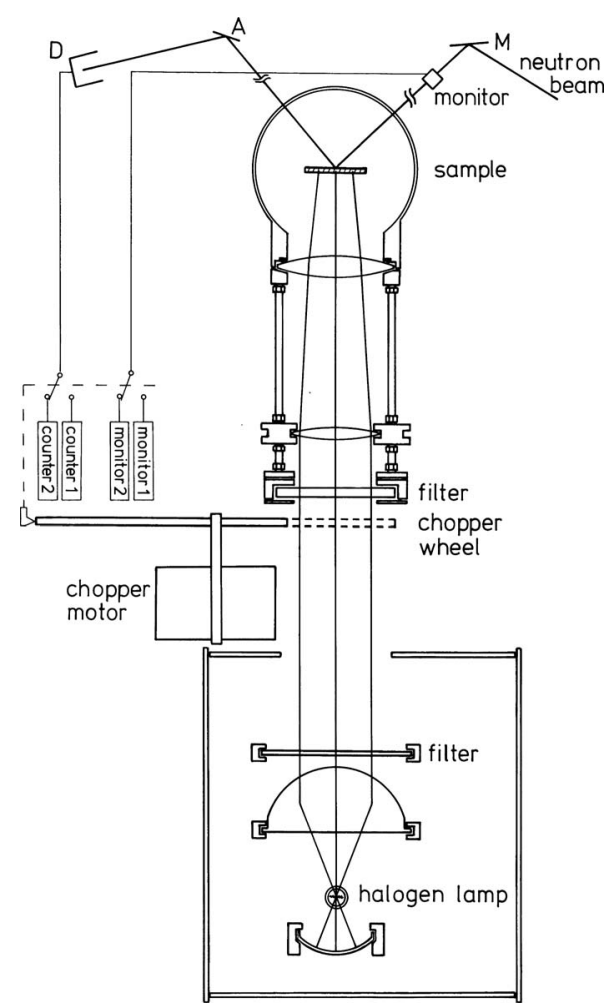

FIG. 1. Conceptual design of the instrumental device developed for simultaneous light irradiation and neutron scattering experiments at low temperatures. M: neutron monochromator; A: neutron analyzer; D: neutron detector.

ments are in good agreement with the results reported in Ref. [5].

Figure 1 shows the instrumental configuration realized for the INS experiments. The light source is a $100 \mathrm{~W}$ halogen lamp (with spectral properties shown in Fig. 2) whose efficiency is optimized by using a reflector. A parallel beam of 5 $\mathrm{cm}$ diameter is created after transmission through a planeconvex lens. Filters of variable bandwidth can be used to yield wavelengths $\lambda$ between 400 and $1000 \mathrm{~nm}$. Figure 2 shows the transmission properties of a broadband filter used for the majority of the experiments. The light is guided to the sample through a lens system whose focal distance can be adjusted to the sample geometry. The sample is mounted in a closed-cycle He refrigerator to achieve temperatures in the range $10 \leq T \leq 300 \mathrm{~K}$. The light beam penetrates the sample chamber through a glass disk or lens inserted into the radiation shield of the cooling system.

In preliminary measurements [8] we failed to discriminate unambiguously photoinduced effects from heating effects since the sample heats up upon illumination, leading to the excitation of additional lattice modes by thermal stimulation. However, heating effects can be avoided by using the pulsed light-beam technique as described in Ref. [9] and shown in Fig. 1. The light beam is periodically interrupted by a rotating wheel with regularly arranged openings; thus, light pulses are created whose frequencies can be varied between $0.05 \leq \nu \leq 5 \mathrm{~Hz}$. The neutron count rates are separately stored for the "light" and "dark" experiments.

A crucial part of the instrumental setup is the sample cell which has to meet simultaneously three different experimental requirements, namely, optimum scattering properties in the INS experiments, optimum efficiency concerning light irradiation, and optimum cooling power. Figure 2 schematically shows the sample container which is a cylinder of 2.5 $\mathrm{cm}$ diameter and $0.15 \mathrm{~cm}$ height. The bottom window is made of aluminum which is transparent for neutrons and guarantees a good heat conduction as well. The top part consists of an optically transparent mica window of $0.07 \mathrm{~cm}$ thickness. An indium ring is used to seal the mica window with the bottom part. The solution is injected into the sample cell through a small hole at the circumference of the cylinder. The temperature is measured in the center of the sample $\left(T_{1}\right)$ as well as at the sample frame $\left(T_{2}\right)$ in order to determine the temperature gradient usually set up under light irradiation. We measured the temperature difference $\Delta T=T_{1}-T_{2}$ between the light and dark experiments with the use of a broadband filter (see Fig. 2) to be less than $0.1 \mathrm{~K}$ for pulse fre-
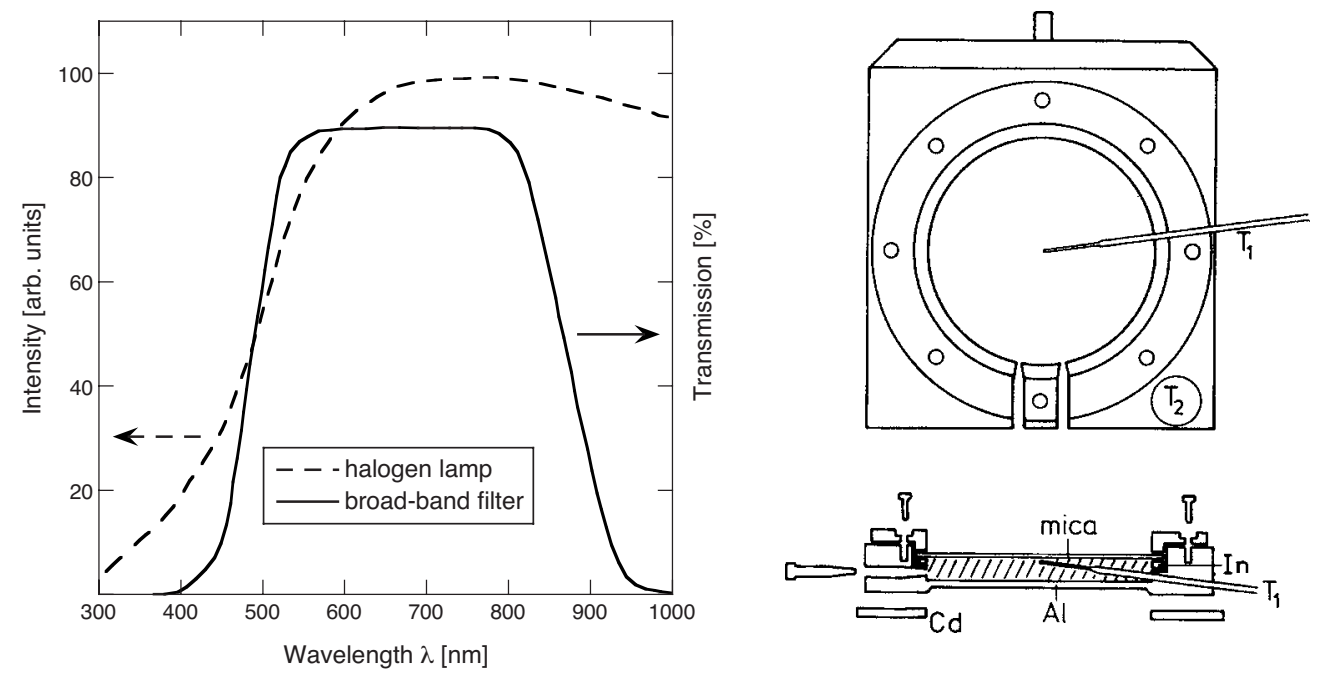

FIG. 2. Left: spectral properties of the halogen lamp and the broadband filter. Right: schematic ground plan and vertical section of the sample cell. 


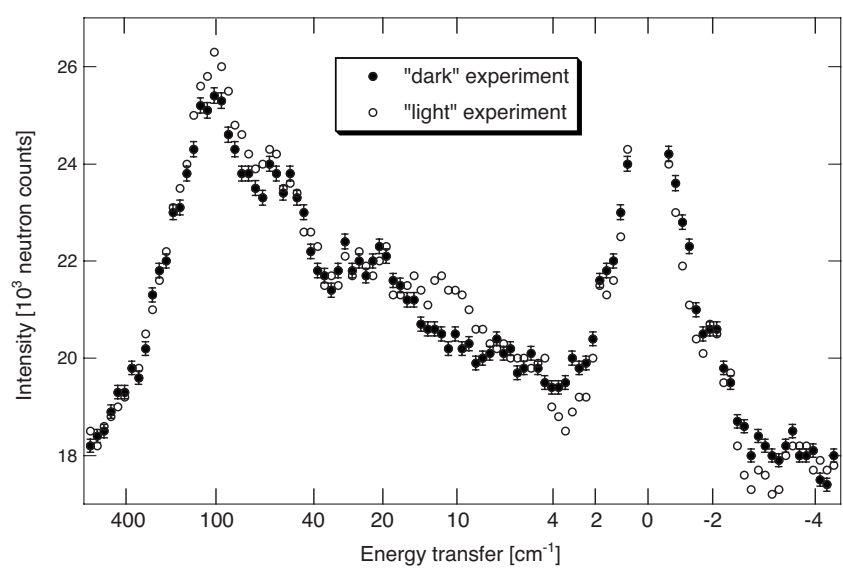

FIG. 3. Neutron time-of-flight spectra measured for a suspension of membranes of the photosynthetic bacterium Rhodopseudomonas viridis in $\mathrm{D}_{2} \mathrm{O}$ buffer at $T=57 \mathrm{~K}$ with incoming neutrons of energy $10.3 \mathrm{~cm}^{-1}$. The pulsed light-beam technique was applied with a pulse frequency $\nu=3 \mathrm{~Hz}$ and with the use of a broadband filter (see Fig. 2). The total counting time amounted to $56 \mathrm{~h}$.

quencies $\nu>0.5 \mathrm{~Hz}$; thus, the photoinduced scattering can be directly obtained from the corresponding intensity difference. Consequently all the experiments were performed with a pulse frequency $\nu=3 \mathrm{~Hz}$.

The INS experiments were carried out with the use of the high-resolution time-of-flight spectrometer IN 5 at the Institute Laue-Langevin, Grenoble. The energy of the incoming neutrons was $10.3 \mathrm{~cm}^{-1}$, giving rise to an energy resolution of $0.3 \mathrm{~cm}^{-1}$ for zero-energy transfer, and the scattered neutrons were detected in a range of scattering angles $92^{\circ} \leq \Phi$ $\leq 134^{\circ}$. The experiments were performed in the reflection geometry as sketched in Fig. 1, i.e., the cylinder axis being parallel to the scattering plane was oriented at an angle $\Phi / 2 \approx 56^{\circ}$.

\section{RESULTS AND DATA ANALYSIS}

Initially we performed the measurements with pseudomonochromatic light of bandwidth $\Delta \lambda=50-80 \mathrm{~nm}$ for wavelengths between 400 and $1000 \mathrm{~nm}$ in order to match particular structural units according to the observed absorption spectrum, but the measured intensity differences between the light and dark experiments were in most cases smaller than the statistical uncertainties of the data. Therefore, the final experiments were carried out in a broad range of wavelengths defined by the broadband filter (see Fig. 2) in order to improve the statistics of the observed signal. The lower cutoff [half height of full maximum (HHFM) $\approx 490 \mathrm{~nm}$ ] largely avoids the stimulation of the carotenoids which also function as light-harvesting units (in addition to the chlorophylls). The upper cutoff (HHFM $\approx 870 \mathrm{~nm})$ was essential to minimize heating of the sample under irradiation, but it still covers the low-wavelength tail of the absorption line of bacteriochlorophyll $\mathrm{b}$ from the antenna complexes.

Representative data sets for $T=57 \mathrm{~K}$ are displayed in Fig. 3. The raw intensity data $I(\Phi, t)$ were transformed from the

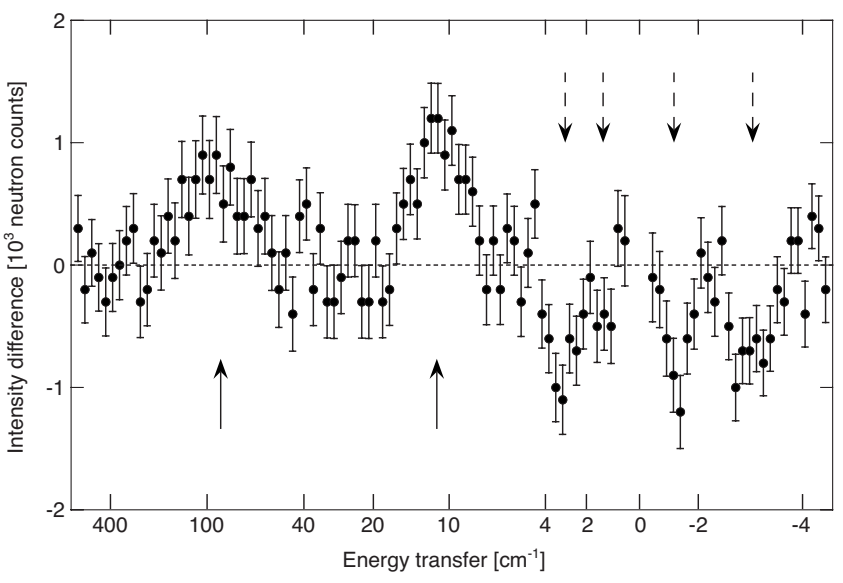

FIG. 4. Intensity difference of the "light" and "dark" energy spectra displayed in Fig. 3. Energy bands corresponding to lightinduced excitations of vibrational modes and freezing of rotational modes are marked with full and broken arrows, respectively.

time scale $t$ to the energy scale $\hbar \omega$ according to the relation [10]

$$
I(\Phi, \omega) \propto I(\Phi, t) t^{4} .
$$

Moreover, the raw data were corrected for background effects and detector efficiency by using the spectra separately measured for the empty sample container and for a vanadium standard, respectively. Equation (1) causes a significant intensity enhancement of the early arriving neutrons, which is intimately connected with a corresponding loss of energy resolution. The observed energy spectra cover both neutron energy-gain and energy-loss processes in which external and internal lattice modes are de-excited and excited, respectively. The energy spectra are therefore related to the generalized density of states of the lattice modes, which exhibits an absolute maximum around an energy transfer of about $100 \mathrm{~cm}^{-1}$. Due to Eq. (1) the energy resolution is typically $10 \mathrm{~cm}^{-1}$ at that energy transfer. Consequently we abstain from presenting the data in equidistant energy units which could easily be achieved by existing interpolation schemes, but we prefer to retain the nonlinear energy scale (corresponding to the original time-of-flight scale) in order not to introduce any artifacts. This is essential when considering intensity differences as discussed below.

There are some relevant differences in the spectra observed with and without light illumination as shown by the intensity difference displayed in Fig. 4. Light irradiation produces an intensity increase at energy transfers centered on $12(2)$ and $88(21) \mathrm{cm}^{-1}$, which are typical for the vibrational states of the sample. On the other hand, we also observe the contrary effect, namely, a decrease in the intensities upon light irradiation at energy transfers around $\pm 1.2(3)$ and $\pm 2.9(5) \mathrm{cm}^{-1}$ as visualized in Fig. 4. Due to Eq. (1) this effect is better resolved in the neutron energy-loss than in the energy-gain part of the spectrum. We argue that these low energies are characteristic of the rotational states of the chlorophyll molecules. Since the chlorophylls in Rhodopseudomonas viridis are bound to $\alpha$ helices in a wheel-like arrangement [6,7] (unlike the light-harvesting 


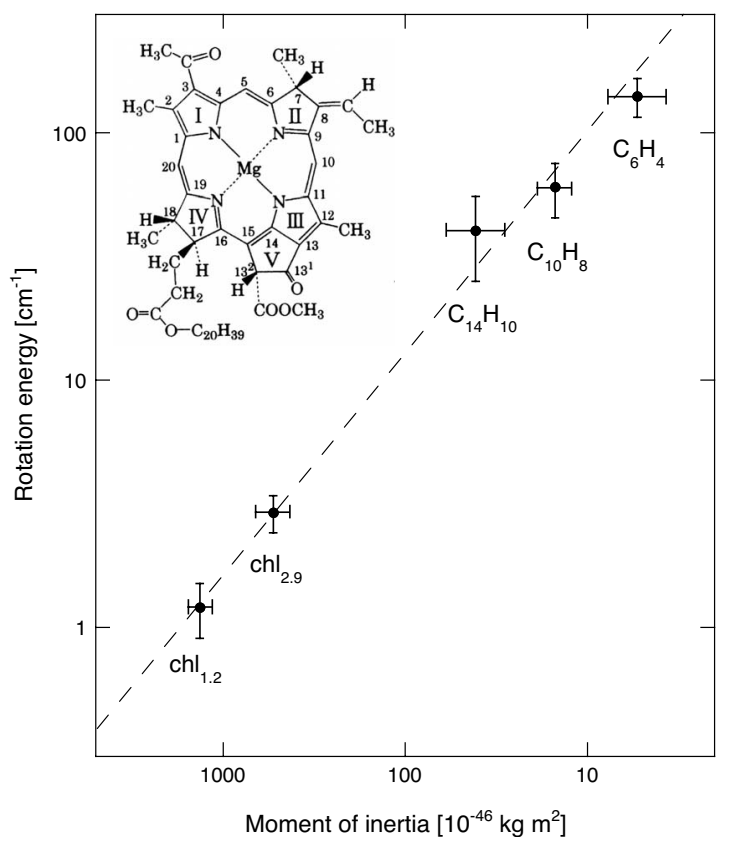

FIG. 5. Rotation energy vs moment of inertia for selected $\mathrm{C}_{n} \mathrm{H}_{m}$ molecules as well as for bacteriochlorophyll b $\left(\mathrm{chl}_{1.2}\right.$ and $\left.\mathrm{chl}_{2.9}\right)$. The dashed line corresponds to the prediction of Eq. (2). The inset shows schematically the porphyrin ring of bacteriochlorophyll $b$ (the phytol chain is summarized by $\mathrm{C}_{20} \mathrm{H}_{39}$ ).

complexes in plants), rotational modes are clearly possible. Because of the absence of literature data we support our hypothesis as outlined below.

The rotational energy of a molecule in free space is [11]

$$
E=J(J+1) \frac{\hbar^{2}}{2 \Theta},
$$

where $J=0,1,2, \ldots$ is the total angular momentum and

$$
\Theta=\sum_{i} m_{i} r_{i}^{2}
$$

is the moment of inertia of the molecule with $r_{i}$ being the perpendicular distance of the $i$ th atom of mass $m_{i}$ from the axis of rotation. As shown in Fig. 5, the experimentally determined rotational energies of some molecules such as benzene $\left(\mathrm{C}_{6} \mathrm{H}_{4}\right)$ [12], naphthalene $\left(\mathrm{C}_{10} \mathrm{H}_{8}\right)$ [13], and anthracene $\left(\mathrm{C}_{14} \mathrm{H}_{10}\right)$ [14] are in good agreement with the proportionality $E \sim 1 / \Theta$ resulting from Eq. (2), as indicated by the dashed line. The error bars of Fig. 5 take account of the anisotropy of the molecules, giving different moments of inertia about the three principal axes of rotation as well as different rotational energies. Figure 5 is extended to include the data for the chlorophyll molecule, which consists of a porphyrin ring and a phytol chain as sketched in the inset. The chlorophyll data are best described if we attribute the excitation at $\pm 1.2(3) \mathrm{cm}^{-1}$ with a rotation of the porphyrin ring around an axis defined by rings I and IV and the phytol chain $\mathrm{C}_{20} \mathrm{H}_{39}$ (see inset of Fig. 5), which yields $\Theta \approx 1.4 \times 10^{-43} \mathrm{~kg} \mathrm{~m}^{2}$, whereas the excitation at $\pm 2.9(5) \mathrm{cm}^{-1}$ can be rationalized by taking the axis of rotation through the central $\mathrm{Mg}^{2+}$ ion with $\Theta \approx 0.5 \times 10^{-43} \mathrm{~kg} \mathrm{~m}^{2}$.

\section{DISCUSSION}

The photoexcited states around 12(2) and $88(21) \mathrm{cm}^{-1}$ may be the result of the electronic-vibrational coupling in the initial steps of photosynthesis. There is a remarkable agreement with low-energy vibrational modes associated with the reaction center of the purple bacterium Rhodobacter capsulatus observed at 15 and $77 \mathrm{~cm}^{-1}$ by multicolor near-infrared femtosecond spectroscopy [15]. This observation that photoexcitation brings about specific vibrational motions in a protein was suggested to be a possible driving force contributing to the very high efficiency of the electron transfer [16]. The similar energies of the two photoinduced vibrational modes in Rhodobacter capsulatus [15] and Rhodopseudomonas viridis (present work) are rather striking.

The intensity decrease of the modes observed at $\pm 1.2(3)$ and $\pm 2.9(5) \mathrm{cm}^{-1}$ means that part of the rotational states is depopulated or "frozen." Freezing of rotational states is a common phenomenon in molecular dynamics [17], but the present experiments provide a neutron spectroscopic observation of light-induced freezing which hinders the Brownian rotation of the chlorophylls and thereby may contribute to an improved efficiency of the photosynthetic process [1].

Although the data obtained with small bandwidth $\Delta \lambda$ $=50-80 \mathrm{~nm}$ are at the limit of statistical relevance, they are interesting in the sense that the light effects appeared to be almost uncorrelated with the optical absorption spectrum. In particular, even for $\lambda \approx 1000 \mathrm{~nm}$, corresponding to the most intense absorption line of bacteriochlorophyll $b$, we observed rather small intensity differences between the light and dark experiments. This indicates that light absorption at $\lambda$ $\approx 1000 \mathrm{~nm}$ occurs with little energy loss through radiationless transitions; thus, it efficiently contributes to the photochemical energy transfer from the antenna complexes to the reaction center.

We also performed INS experiments at room temperature; however, we could not detect relevant differences between the light and dark measurements. This is certainly due to the fact that the lattice modes become increasingly damped when raising the temperature. A similar observation was also reported in Ref. [15]. Finally, in order to exclude any artifacts, INS experiments were carried out for a suspension of membranes which did not contain photosynthetic units. As expected, light illumination had no effect on the observed energy spectra. This reinforces our interpretation that the photoinduced effects have to be attributed to the chlorophyll molecules.

\section{CONCLUDING REMARKS}

With these exploratory INS experiments we have introduced a technique which is capable to measure the lightinduced increase and decrease in the population of vibrational and rotational states in chlorophyll-protein systems, respectively. A high efficiency of photochemical energy transfer requires that the radiationless transitions are minimized. A measurement of the irradiation effect observed by neutron spectroscopy may therefore be a useful tool to investigate where, and how much, energy is "lost" in the initial steps of photosynthesis. 
We are aware that our present interpretation of the observed light-induced effects is on a rather qualitative level, but it is extremely difficult to calculate the vibrational and rotational energies of such a complicated object as the photosynthetic unit, particularly within its natural environment of a membrane structure. However, in forthcoming experiments one can envisage the use of a quasioriented system by applying a high magnetic field [18] as well as illumination in a narrow wavelength band to aid in a more detailed assignment of vibrational and rotational states to individual structural units. The latter experiments will certainly become fea- sible with the advent of third-generation spallation neutron sources, which are already built in the USA, under construction in Japan, and in the planning state in Europe.

\section{ACKNOWLEDGMENTS}

We are indebted to W. Stark of the Institute for Cell Biology, ETH Zurich, for preparing and characterizing the samples, and to W. Langel of the ILL Grenoble for experimental assistance in the present work.
[1] W. Junge and A. Eckhof, FEBS Lett. 36, 207 (1973).

[2] H. N. Bordallo, N. Barthes, and J. Eckert, Physica B 241-243, 1138 (1998).

[3] J. Pieper, A. Buchsteiner, N. A. Dencher, R. E. Lechner, and T. Hauss, Phys. Rev. Lett. 100, 228103 (2008).

[4] B. Jacrot, S. Cusack, A. J. Dianoux, and D. M. Engelman, Nature (London) 300, 84 (1982).

[5] F. Jay, M. Lambillotte, W. Stark, and K. Mühlethaler, EMBO J. 3, 773 (1984).

[6] W. Stark, W. Kühlbrandt, I. Wildhaber, E. Wehrli, and K. Mühlethaler, EMBO J. 3, 777 (1984).

[7] J. Deisenhofer, O. Epp, K. Miki, R. Huber, and H. Michel, Nature (London) 318, 618 (1985).

[8] A. Furrer, A. Stöckli, W. Hälg, W. Kühlbrandt, K. Mühlethaler, and E. Wehrli, Helv. Phys. Acta 56, 655 (1983).

[9] A. Stöckli, A. Isacson, M. Koch, and A. Furrer, Neutron Scattering in the 'Nineties (International Atomic Energy Agency, Vienna, 1985), p. 199.

[10] A. Furrer, J. Mesot, and Th. Strässle, Neutron Scattering in
Condensed Matter Physics (World Scientific, Singapore, 2009).

[11] P. W. Atkins, Physical Chemistry (Oxford University Press, Oxford, 1998).

[12] B. H. Meier, R. Meyer, R. R. Ernst, P. Zolliker, A. Furrer, and W. Hälg, Chem. Phys. Lett. 103, 169 (1983).

[13] I. Natkaniec, E. L. Bokhenkov, B. Dorner, J. Kalus, G. A. Mackenzie, G. S. Pawley, U. Schmelzer, and E. F. Sheka, J. Phys. C 13, 4265 (1980).

[14] B. Dorner, E. L. Bokhenkov, S. L. Chaplot, J. Kalus, I. Natkaniec, G. S. Pawley, U. Schmelzer, and E. F. Sheka, J. Phys. C 15, 2353 (1982).

[15] M. H. Vos, F. Rappaport, J. C. Lambry, J. Breton, and J. L. Martin, Nature (London) 363, 320 (1993).

[16] J. Maddox, Nature (London) 363, 297 (1993).

[17] H. Boutin and S. Yip, Molecular Spectroscopy with Neutrons (MIT Press, Cambridge, MA, 1968).

[18] D. M. Sadler and D. L. Worcester, J. Mol. Biol. 159, 467 (1982). 